Efecto de agentes estabilizantes en jugo de piña (Ananas comosus) y coco (Cocos nucifera $L$.) edulcorado

\author{
Brayan Castulovich $\odot$, Jaruvy Franco \\ Universidad Tecnológica de Panamá \\ castulovich.b@gmail.com; jaruvy.f@outlook.es \\ DOI https://doi.org/10.33412/pri.v9.1.2063
}

\section{(2)(1) $\$(0)$}

Resumen: En esta investigación se realizó una evaluación de los agentes estabilizantes en un jugo de piña y coco edulcorado, para mejorar la inestabilidad coloidal que se presentan en los jugos mixtos de frutas. Se contempló una muestra control, la cual no contaba con estabilizantes, siendo esta la que presentaba las separaciones de fases que es el problema que se observa con los jugos en el mercado actual. Se formularon dos muestras con diferentes estabilizantes, la formulación 1 se estabilizó a una concentración de $0.024 \%$ de goma xantana y $0.015 \%$ carboximetilcelulosa y la formulación 2 se estabilizó a una concentración de $0.024 \%$ de goma xantana y $0.015 \%$ carragenina, con el fin de corregir este defecto de calidad. Las propiedades fisicoquímicas evaluadas fueron: acidez total, sólidos solubles, carbohidratos totales, cenizas, grasas, humedad, proteinas, donde se encontraron diferencias significativas. Se evaluó la acidez total titulable, densidad y sólidos solubles donde no se observaron cambios entre las muestras. El porcentaje de sinéresis, muestra valores de separación altos en ambas formulaciones con respecto al control. Los valores de la viscosidad obtenido a temperaturas de $15^{\circ}, 35^{\circ}$ y $50^{\circ} \mathrm{C}$ varian significativamente, resultando el coeficiente de consistencia $K$ alterado, debido a una menor consistencia y/o resistencia al flujo del jugo con las temperaturas. La adición de estabilizantes cambia la composición proximal y reológicas del jugo de piña y coco. Para obtener mejores cambios se debe utilizar porcentajes más altos de hidrocoloides (1-3\%), para que la sinéresis sea menor y no se logren apreciar la separación de fases.

Palabras clave: jugo, piña, coco, sucralosa, goma xantana, carragenina, carboximetilcelulosa.

Title: Effects of stabilizing agents in pineapple (Ananas comosus) and coconut (Cocos nucifera L.) juice sweetened.

Abstract: In this investigation an evaluation of the stabilizing agents in a pineapple and sweetened coconut juice was made to improve the colloidal instability that occur in mixed fruit juices. A control sample was contemplated which did not have stabilizers, this being the one that presented the phase separations that is the problem observed with the juices in the current market. Two samples were formulated with different stabilizers, formulation 1 was stabilized at a concentration of $0.024 \%$ xanthan gum and $0.015 \%$ carboxymethylcellulose and formulation 2 was stabilized at a concentration of $0.024 \%$ xanthan gum and $0.015 \%$ carrageenan to correct this quality defect. The physicochemical properties evaluated were: total acidity, soluble solids, total carbohydrates, ash, fat, moisture, proteins where significant differences were found. The titratable total acidity, density and soluble solids were evaluated where no changes were observed between the samples. The percentage of syneresis shows high separation values in both formulations with respect to the control. The values of the viscosity obtained at temperatures of $15^{\circ}, 35^{\circ}$ and $50^{\circ} \mathrm{C}$ vary significantly, resulting in the coefficient of consistency $\mathrm{K}$ altered due to a lower consistency and/or resistance to the flow of the juice with the temperatures. The addition of stabilizers changes the proximal and rheological composition of pineapple and coconut juice. To obtain better changes, higher percentages of hydrocolloids (1-3\%) should be used so that the syneresis is lower and phase separation cannot be appreciated.

Key words: juice, pineapple, coconut, sucralose, xanthan gum, carrageenan, carboxymethyl cellulose.

Tipo de artículo: original

Fecha de recepción: 21 de julio de 2018

Fecha de aceptación: 17 de octubre de 2018

\section{Introducción}

La piña (Ananas comosus) y coco (Cocos nucifera L.) son frutas tropicales preferidas por los consumidores por su valor nutritivo y fuente de antioxidantes. El desarrollo de jugos a base de piña y coco son comúnmente elaborados y comercializados de forma individual y muy poco de forma mixta. Esto se debe al comportamiento de los componentes, ya que, estas frutas están constituidas por materiales poliméricos insolubles cargados negativamente, que en dispersión afectan la estabilidad coloidal de partículas, favoreciendo a la inestabilidad y provocando la separación de fases durante el almacenamiento [1]. Siendo este el principal problema de la elaboración de los jugos mixtos de frutas, porque la inestabilidad coloidal en suspensiones alimentarias se percibe como defecto de calidad, afectando la aceptabilidad y comercialización del producto [2].

Los agentes estabilizantes como la carragenina (E407), goma xantana (E415) y carboximetilcelulosa (E466) son sustancias hidrofilicas que son utilizadas para estabilizar suspensiones, debido a su efecto sobre la viscosidad, evitando procesos de separación de fases en los jugos durante el almacenamiento [3]. Se ha evidenciado que al incorporar hidrocoloides a un producto líquido aumentan la turbidez, controlan la sedimentación de partículas, incrementan la viscosidad de la suspensión, sin afectar las propiedades fisicoquímicas y sensoriales de las bebidas. Este incremento de la viscosidad se puede evidenciar por el comportamiento reológico del alimento, mientras que el efecto de 
las propiedades fisicoquímicas mediante análisis gravimétrico y proximal [4].

En el estudio realizado por Londoño, Lucas y Quintero, al utilizar $0.02 \%$ de pectina, $0.015 \%$ de carboximetilcelulosa y $0.014 \%$ de goma xantana, logran estabilizar el jugo de naranjada recién exprimido. Estos estabilizantes aseguran una mínima separación de fases y una viscosidad constante de 5,2 cP durante el almacenamiento, es por ello que en esta investigación se utilizó esta mezcla como referencia para formulación del jugo de piña y coco, modificando la mezcla de estabilizante, empleando en $0.014 \%$ carboximetilcelulosa y $0.024 \%$ goma xantana, otro aporte fue comparar esa formulación con una nueva a la cual solo se le modificó el estabilizante carboximetilcelulosa por carragenina con las mismas concentraciones, que también es un estabilizante que se puede utilizar en jugo y se ha evidenciado efectos positivos al ser adicionados en líquidos, actuando como espesante y no permite la gelificación (e.g., [5], [7], [11]).

El objetivo de este estudio fue evaluar los cambios reológicos y fisicoquímicos en el jugo de piña y coco por la adición de diferentes mezclas de estabilizantes.

\section{Agentes estabilizantes}

Los estabilizantes son macromoléculas, principalmente polisacáridos (coloides, hidrocoloides y gomas), que mantienen 0 mejoran la estructura de los alimentos y hacen posible la distribución fina y unitaria de las partículas que no son solubles entre sí [5].

\subsection{Carboximetilcelulosa}

La carboximetilcelulosa (E466) se origina de celulosa purificada a partir de algodón y pulpa de madera, la cual se modifica químicamente sustituyendo los hidrógenos por grupos carboximetilos. En su estructura contiene un grupo carboxílico hidrofílico que aumenta su solubilidad en el agua. No necesita calentamiento para su hidratación, funciona como un estabilizador fuerte y solo se necesita en una proporción de $0,1 \%$ a $0,2 \%$ en la mezcla.

Toxicológicamente no está enlistado como cancerígeno por la NTP (National Toxicology Program); no está regulado como cancerígeno por la OSHA (Occupational Safety \& Health Administration) y no está evaluado por la IARC (International Agency for Research on Cáncer). Un único caso de dermatitis alérgica al contacto se reporta después de sostener contacto repetido durante un período largo (8 años) con CMC purificada [5].

\subsection{Goma xantana}

La goma xantana (E415) se produce por la bacteria Xanthomonas campestris. Es un polisacárido conformado por una cadena de glucosa que presenta ramificaciones de trisacáridos laterales. Se solubiliza en agua fría y es capaz de hidratarse rápidamente una vez se ha dispersado por toda la mezcla. Es resistente a los cambios de temperatura y $\mathrm{pH}$. Brinda propiedades pseudoplásticas a la mezcla y se diluye con facilidad al aplicar un esfuerzo cortante.
El DL50 es de 45000 mg/Kg. No se dispone de información sobre ningún componente de este producto, que presente niveles mayores o iguales que $0,1 \%$, como carcinógeno humano probable, posible o confirmado por la IARC (Agencia Internacional de Investigaciones sobre Carcinógenos). No provoca lesiones cutáneas ni oculares al resultar ser no irritante [5].

\subsection{Carragenina}

Los carragenanos (E407) son polisacáridos complejos cuyos principales constituyentes son D-galactopiranosa y 3,6-anhidro-Dgalactosa, esterificados con $\mathrm{H}_{2} \mathrm{SO}_{4}$, derivados originalmente de algas marinas rojas de la clase Rhodophycae, tales como Chondrus crispus, Kappaphycus alverezii y Eucheuma denticulaturn.

La carragenina está disponible en varios tipos, los más comunes son kappa (I y II), iota (i) y lambda $(\lambda)$. Carragenina Lambda $(\lambda)$. Es la más soluble en agua y leche, ya que posee un contenido de un $35 \%$ de éster sulfato y un $0 \%$ de 3,6 anhidrogalactosa, lo que imposibilita la gelificación. Imparte alta viscosidad a los sistemas, no requiere de calentamiento para su hidratación.

Este producto no presenta peligro de toxicidad crónica basado en la información conocida o suministrada. No se conocen efectos mutagénicos o teratogénicos. No contiene ingredientes listados como carcinógenos. La aspiración puede provocar neumonía química. La inhalación excesiva de polvo puede impedir mecánicamente la respiración [5].

\section{Metodología}

Para el desarrollo de esta investigación se adquirió la materia prima e ingredientes en tiendas de la localidad para proceder con la elaboración, caracterización fisicoquímica y reológica del jugo procesado.

\subsection{Preparación del jugo}

La piña y el coco se obtuvieron en estado de madurez comercial, sanos, libres del ataque de hongos, a los cuales, se retiró la cáscara. Se le realizó un despulpado a la piña (TEREYMAG) con una malla de tamaño de poro de $2 \mathrm{~mm}$ y aparte se procesó (BLACK \& DECKER) el coco con agua para extraer la pulpa, para luego ser filtrada y mezclada con la pulpa de piña para obtener jugo control en una proporción de $54 \%$ piña, $23 \%$ coco, $23 \%$ agua. El jugo obtenido se usó para formular dos mezclas con diferentes estabilizantes, la formulación 1 se estabilizó a una concentración de $0.024 \%$ de goma xantan y $0.015 \%$ carboximetilcelulosa y la formulación 2 se estabilizó a una concentración de $0.024 \%$ de goma xantana y $0.015 \%$ carragenina y el control al cual no se le agrego ningún estabilizante, las cuales fueron adaptadas de la fuente [7]. En ambas formulaciones y al control se le adicionó $1000 \mathrm{mg}$ de sucralosa adaptada de la fuente [8], $187 \mathrm{mg}$ de ácido ascórbico y $10 \mathrm{mg}$ de ácido cítrico, los cuales se encuentra dentro de los valores diarios recomendados [6]. El jugo se pasteurizó durante 15 segundos a $85^{\circ} \mathrm{C}$, luego se sometió a enfriamiento y posteriormente se introdujo en botellas de vidrio, sellado al vacío para ser almacenado [7]. 


\subsection{Pruebas fisicoquímicas}

Al jugo de piña y coco se le realizó pruebas fisicoquímicamente por duplicado. Los parámetros analizados fueron: carbohidratos totales por espectrofotometría UV (SPECTRONIC GENESYS 5), se determinaron por el método del fenol-ácido sulfúrico que consiste en la deshidratación por reacción con ácido sulfúrico para producir derivados de furfural. Es un método colorimétrico que consiste en preparar una solución acuosa de $2 \mathrm{~mL}$ de jugo y $1 \mathrm{~mL}$ de fenol $5 \%$, se adiciona $5 \mathrm{~mL}$ de $\mathrm{H}_{2} \mathrm{SO}_{4}$, se agita y se deja reposar por $10 \mathrm{~min}$. Se vuelve agitar por 30 segundos y se colocan durante 20 minutos en un baño de agua a temperatura ambiente, luego, la absorción de luz a $490 \mathrm{~nm}$ se registra en un espectrofotómetro (SPECTRONIC GENESYS 5), la cuantificación se realizó utilizando una curva patrón de glucosa [17]. Humedad por el método AOAC 20.013. para jugo de frutas con alto contenido de azúcar. Proteínas crudas por el método Kjeldahl AOAC 2001.11., mediante las tres etapas: digestión (SPEED DIGESTER K-425)-destilación (BUCHI Destilación Unit $\mathrm{K}-350$ )-titulación con hidróxido de sodio 0.1M. La cuantificación de proteína se calculó por el volumen consumido de hidróxido de sodio y factor de correlación de 0.6 (fórmula 3 y 4). Grasas totales se determinó por el método de Bligh y Dyer, por medio de extracción-húmeda con cloroformo-metanol, para alimento con alto contenido de humedad. Las cenizas, según método AOAC 923.03. por calcinación a $550^{\circ} \mathrm{C}$, hasta la obtención de cenizas blancas. Los sólidos solubles con un refractómetro de mesa (VEE GEE BX-50), escala de Brix 0 a $50 \%$, siguiendo el método AOAC 932.12. La acidez titulable por el método AOAC 939.05. expresado como porcentaje de ácido cítrico. El pH por el método potenciométrico, mediante un potenciómetro con electrodo de vidrio (MP511 Lab pH Meter), según el método AOAC 981.12. La densidad aparente se determinó por el método del picnómetro Gay-Lussac donde se emplea un picnómetro de volumen constante $25 \mathrm{ml} \mathrm{a} 20^{\circ} \mathrm{C}$ utilizando agua destilada como líquido de referencia y la densidad de los líquidos según el método AOAC 945.06. El calor específico se estimó por medio de la fracción de masa de sus componentes: agua, proteínas, grasas, carbohidratos y cenizas (fórmula 1). La sinéresis fue medida estableciendo la relación entre el volumen separado y el volumen total del tubo graduado que contenía el jugo de piña y coco (fórmula 2), esto se evaluó por centrifugación a 3000 RPM por 10 minutos (DYNAC), (e.g., [9], [10], [11]).

$$
\begin{aligned}
& \mathrm{cp}=4.180 \mathrm{X}_{\text {water }}+1.547 \mathrm{X}_{\text {prot }}+1.672 \mathrm{X}_{\text {fat }}+1.42 \mathrm{X}_{\mathrm{CHO}}+0.836 \mathrm{X}_{\text {ash }} \\
& \text { sinéresis, } \%=\text { volumen separado } \times 100 \\
& \text { volumen total }
\end{aligned}
$$

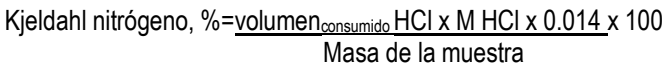

$$
\begin{aligned}
& \text { Proteína, } \%=\% \text { Kjeldahl }{ }^{*} \mathrm{~F}
\end{aligned}
$$

Los resultados fueron analizados mediante ANOVA con un nivel de significancia del $5 \%$, se aplicó el test de Tukey para comparación de medias, utilizando Excel para el procesamiento de los datos.

\subsection{Pruebas reológicas}

El comportamiento reológico fue determinado utilizando un viscosímetro rotacional (FUNGILAB) con el husillo R4, el viscosímetro tiene una resolución de $0,1{ }^{\circ} \mathrm{C}\left(0,1722{ }^{\circ} \mathrm{F}\right)$ y precisión: $\pm 0,1{ }^{\circ} \mathrm{C}$. El viscosímetro rotacional se usa para describir la consistencia de diferentes productos y normalmente es definida por dos componentes: viscosidad y elasticidad.

\section{Resultado y discusión}

Cada una de las pruebas realizadas a continuación se hicieron para comprobar los efectos de los estabilizantes y cuantificar los cambios que ocasionan cada uno de ellos en la muestra.

\subsection{Análisis proximal}

El efecto que tienen los estabilizantes sobre la composición química del producto del jugo, muestra que hay cambios leves $(P \leq 0.05)$, en la humedad, grasas, proteínas y carbohidratos de ambas formulaciones frente al control, debido a la función que tienen los estabilizantes de ligar (tabla 1). Los estabilizantes ocasionaron cambios en las muestras, puesto que la interacción sinérgica entre las combinaciones de hidrocoloides de las formulaciones fue deficiente. Este suceso no debería presentarse, ya que los estabilizantes utilizados en combinaciones correctas y aprovechando las acciones sinérgicas entre ellos, suelen proporcionar estabilidad frente a influencias físicas, químicas y biológicas (e.g., [12], [13]).

Tabla 1. Composición proximal

\begin{tabular}{|c|c|c|c|}
\hline Pruebas & Control & Formulación 1 & Formulación 2 \\
\hline Humedad (\%) & $88.72 \pm 0.085^{\mathrm{a}}$ & $87.46 \pm 0.29^{\mathrm{b}}$ & $87.23 \pm 0.10^{\mathrm{c}}$ \\
\hline Carbohidrato (\%) & $8.12 \pm 0.28^{\mathrm{a}}$ & $8.11 \pm 0.098^{\mathrm{a}}$ & $8.45 \pm 0.32^{\mathrm{b}}$ \\
\hline Grasa (\%) & $2.10 \pm 0.098^{\mathrm{a}}$ & $3.24 \pm 0.036^{\mathrm{b}}$ & $3.28 \pm 0.127^{\mathrm{b}}$ \\
\hline Proteína (\%) & $0.76 \pm 0.012^{\mathrm{a}}$ & $0.92 \pm 0.24^{\mathrm{b}}$ & $0.84 \pm 0.001^{\mathrm{c}}$ \\
\hline Ceniza (\%) & $0.25 \pm 0.056^{\mathrm{a}}$ & $0.33 \pm 0.064^{\mathrm{b}}$ & $0.30 \pm 0.050^{\mathrm{b}}$ \\
\hline
\end{tabular}

Promedios con letras distintas, entre filas, indican diferencia significativa según la prueba de Tukey $(\mathrm{P} \leq 0,05)$.

\subsection{Análisis fisicoquímico}

Los resultados de los análisis fisicoquímicos no presentan cambios significativos ( $\mathrm{P} \leq 0.05)$, en acidez, $\mathrm{pH}$ y sólidos solubles totales (tabla 2), ya que los hidrocoloides tienen excelente estabilidad en un rango amplio de $\mathrm{pH}$, logrando así no variar estas propiedades en el jugo. En cuanto a la acidez y los sólidos solubles totales, no varían al adicionar hidrocoloides, puesto que las características de los estabilizantes empleados no afectan estas propiedades fisicoquímicas (e.g., [12], [13]). 
Tabla 2. Análisis fisicoquímico

\begin{tabular}{|c|c|c|c|}
\hline Pruebas & Control & Formulación 1 & Formulación 2 \\
\hline Acidez (\% ácido. cítrico) & $0.2 \pm 0.001^{\mathrm{a}}$ & $0.2 \pm 0.001^{\mathrm{a}}$ & $0.2 \pm 0.001^{\mathrm{a}}$ \\
\hline $\mathrm{pH}$ & $5.31 \pm 0.039^{\mathrm{a}}$ & $5.32 \pm 0.012^{\mathrm{a}}$ & $5.28 \pm 0.010^{\mathrm{a}}$ \\
\hline $\begin{array}{c}\text { Sólidos solubles totales } \\
\left({ }^{\circ} \text { Brix) }\right.\end{array}$ & $8 \pm 0.001^{\mathrm{a}}$ & $8 \pm 0.001^{\mathrm{a}}$ & $8 \pm 0.001^{\mathrm{a}}$ \\
\hline
\end{tabular}

Promedios con letras distintas, entre filas, indican diferencia significativa según la prueba de Tukey $(\mathrm{P} \leq 0.05)$.

Los resultados de calor específico y densidad, las formulaciones 1 y 2 con respecto al control, presentaron diferencias significativas $(P \leq 0,05)$. Estos resultados pueden ser utilizados para modelar la transferencia de calor y masa, que es útil al dimensionar equipo y estandarizar el proceso de la elaboración del jugo (tabla 3) [16].

Tabla 3. Calor específico y densidad

\begin{tabular}{|c|c|c|c|}
\hline Pruebas & Control & Formulación 1 & Formulación 2 \\
\hline Calor específico $(\mathrm{J} / \mathrm{Kg} \mathrm{K})$ & $3.87^{\mathrm{a}}$ & $3.84^{\mathrm{b}}$ & $3.84^{\mathrm{b}}$ \\
\hline Densidad $\left(\mathrm{Kg} / \mathrm{m}^{3}\right)$ & $1036 \pm 1^{\mathrm{a}}$ & $1043 \pm 1^{\mathrm{b}}$ & $1041 \pm 0.001^{\mathrm{b}}$ \\
\hline
\end{tabular}

Promedios con letras distintas, entre filas, indican diferencia significativa según la prueba de Tukey $(\mathrm{P} \leq 0,05)$.

El porcentaje de sinéresis muestra valores de separación altos en ambas formulaciones con respecto al control, esto nos indica que se da una separación de fase durante un periodo de tiempo hasta que se da el equilibrio (figura 1). En cuanto a la formulación 1 , aunque presentó una baja sinéresis con respecto al control, posiblemente se debió a que la goma no interactuó sinérgicamente con el hidrocoloide E466, indicando que no tiene la capacidad de estabilizar completamente el sistema. En la formulación 2, se obtuvo una menor sinéresis, esto se le atribuye a que la goma xantana actúa sinérgicamente con la carragenina. También, por habilidad para ligar toda el agua restante en soluciones acuosas, lo que es importante para controlar la reología del sistema, ya que estabilizan las emulsiones y suspensiones de partículas, controlan la cristalización e inhiben la sinéresis (tabla 4), (e.g., [11], [12]).

Tabla 4. Sinéresis

\begin{tabular}{|c|c|c|c|}
\hline Prueba & Control & Formulación 1 & Formulación 2 \\
\hline Sinéresis (\%) & $18.12 \pm 0.881^{\mathrm{a}}$ & $13.75 \pm 1.77^{\mathrm{b}}$ & $9.3 \pm 0.884^{\mathrm{c}}$ \\
\hline
\end{tabular}

Promedios con letras distintas, entre filas, indican diferencia significativa según la prueba de Tukey $(\mathrm{P} \leq 0,05)$.

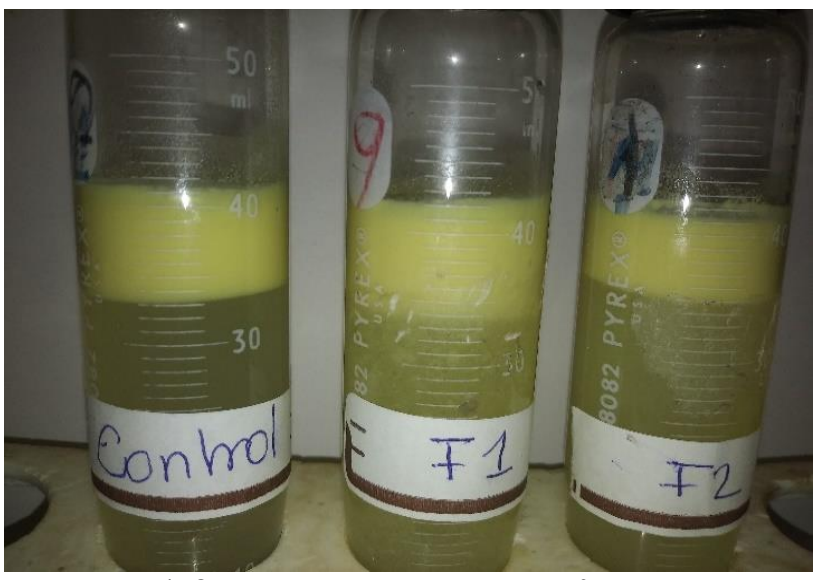

Figura 1. Sinéresis del control y las dos formulaciones.

\subsection{Comportamiento reológico}

La adición de hidrocoloides afecta el índice de comportamiento al flujo y el coeficiente de consistencia, por lo tanto, la viscosidad. La adición o aumento en la concentración de hidrocoloides disminuye el índice de comportamiento al flujo, por lo cual los jugos se hacen más seudoplásticos. Este comportamiento se puede atribuir a que mejora la interacción partícula-partícula, que ocasiona una mejor orientación de las partículas, y aumenta el coeficiente de consistencia. Cabe destacar que la adición de hidrocoloides como el E466, E415 y E407, alteran la viscosidad de la fase continua en jugos mejorando la estabilidad de partículas en dispersión. También, la ley de Stokes ilustra que el fenómeno de precipitación de partículas es inverso a la viscosidad del medio, y que una mayor consistencia de la fase continua puede disminuir la separación de fases y conferir estabilidad estérica en suspensiones alimentarias [1].

Los valores de la viscosidad obtenido a diferentes temperaturas varían significativamente $(P \leq 0,05)$ por la adición de la mezcla estabilizantes, y por lo tanto el coeficiente de consistencia $\mathrm{K}$. Esto se puede afirmar debido a que $\mathrm{K}$ disminuye con la temperatura, por ello tiene el mismo comportamiento que la viscosidad (tabla 5). Esto se explica porque la temperatura es una medida indirecta de la energía interna, que al incrementar se reducen las fuerzas intermoleculares, ocasionando su distanciamiento y facilitando el movimiento y la vibración, lo que induce a una menor consistencia y/o resistencia al flujo. Como la viscosidad varía en función de la temperatura, el jugo de piña y coco es un fluido no newtoniano (e.g., [14], [15]).

Tabla 5. Viscosidad con respecto a la temperatura

\begin{tabular}{|l|c|c|c|}
\hline \multicolumn{4}{|c|}{ Viscosidad (cP) } \\
\hline Temperatura & $15^{\circ} \mathrm{C}$ & $35^{\circ} \mathrm{C}$ & $50^{\circ} \mathrm{C}$ \\
\hline Control & $17.79^{\mathrm{a}}$ & $12.24^{\mathrm{a}}$ & $11.73^{\mathrm{a}}$ \\
\hline Formulación 1 & $19.08^{\mathrm{b}}$ & $13.36^{\mathrm{b}}$ & $11.89^{\mathrm{b}}$ \\
\hline Formulación 2 & $21.18^{\mathrm{c}}$ & $14.95^{\mathrm{c}}$ & $11.32^{\mathrm{c}}$ \\
\hline
\end{tabular}

Promedios con letras distintas, en la misma columna, indican diferencia significativa según la prueba de Tukey $(P \leq 0,05)$. 


\section{Conclusión}

La adición de hidrocoloides E466, E415 y E407, cambia levemente la composición proximal y reológicas del jugo de piña y coco. Para el jugo, la mejor mezcla de estabilizante fue de $0.024 \%$ de goma xantana y $0.015 \%$ carragenina.

La sinergia entre hidrocoloides es afectada por la concentración y el producto utilizado. Para obtener mejores cambios se debe utilizar porcentajes más altos de hidrocoloides (1-3\%) para que la sinéresis sea menor y no se logren apreciar la separación de fases. Esto se debe realizar teniendo en cuenta que a mayores concentraciones de estabilizantes el efecto de gelatinización podría afectar.

El jugo de piña y coco tiene un comportamiento no newtoniano, esto se evidencia porque la viscosidad cambia en función de la temperatura y se obtiene una mayor viscosidad en la formulación 2.

\section{Referencias}

[1] E. Lozano, J. Figueroa, J. Salcedo, R. Torres, R. Andrade. "Efecto de la adición de hidrocoloides en el comportamiento reológico de néctar mixto", Agronomía Colombiana, vol. 34, pp. S464-S466, 2016.

[2] J. Figueroa, C. Márquez, H. Ciro. "Evaluación de estabilidad coloidal en bebidas de tomate de árbol". Agronomía Colombiana, vol. 34. pp. S792-S795, 2016.

[3] M. Quek, N. Chin, Y. Yusof, "Modelling of rheological behaviour of soursop juice concentrates using shear rate-temperature-concentration superposition", Journal of Food Engineering, vol. 118, pp. 380-386, 2013.

[4] G. Ibrahim, I. Hassan, A. Abd-Elrashid, K. El-Massry, A. Eh-Ghorab. M. Ramadan, F. Osman. Effect of clouding agents on the quality of apple juice during storage. Food Hydrocolloids, vol. 25, pp.91-97, 2011.
[5] S. Jaimes, J. Ramírez, A. Rodríguez. Estabilizantes más utilizados en helados. Heladería Panadería Latinoamericana, vol. 251, pp. 66-75, 2017.

[6] Reglamento Técnico Centroamericano (RTC), Alimentos y bebidas procesados. Néctares de frutas. Especificaciones, RTCA 67.04.48:07, 2007.

[7] M. Londoño, J. Lucas J, V. Quintero. "Estudio de la viabilidad del Lactobacillus casei en jugo de naranja (Citrus sinensis) adicionado con vitamina $\mathrm{C}$, Calcio y oligofructosa", Ciencia e Ingeniería, vol. 18, pp. 31-38, 2015.

[8] V. Quitral, A. Pinheiro, C. Carrera, G. Gallo, P. Moyano, J. Salinas, P. Jiménez. "Efecto de edulcorantes no calóricos en la calidad sensorial de jugo de naranja", Revista Chile Nutrición, vol. 42, pp. 77-82, 2015.

[9] A.O.A.C. 1980. Official methods of Analysis. 16 th Edition. Association of official Analytycal Chemists. Washington D.C; USA.

[10] A.O.A.C. 2000. Official methods of Analysis.16 th Edition. Association of official Analytycal Chemists. Washington D.C; USA.

[11] H. Súarez, K. Cabrera, D. Molina, J. Sepulveda, M. "Ospina. Influencia de goma xantan y goma guar sobre las propiedades reológicas de leche saborizada con cocoa", Biotecnología en el Sector Agropecuario y Agroindustrial, vol. 10, pp. 51-59, 2012.

[12] J. Ampuero, "Efecto de la concentración de tres gomas sobre el indice de consistencia y la sinéresis de la salsa de aji," M. Eng. tesis, Univesidad San Ignacio de Loyola, Perú, 2016.

[13] H. Schmidt-Hebbel, Avances en aditivos alimentarios y la reglamentación de los alimentos, Editorial Universitaria, Santiago-Chile, 1990.

[14] E. Arroyo, K. Contreras, J. Figueroa. "Propiedades reológicas de pulpa edulcorada de tomate de árbol (Cyphomandra betacea)", Agronomía Colombiana, vol. 34, pp. S419-S421, 2016.

[15] V. Falguera, A. Ibarz. "Juice processing: Quality, safety and value-added opportunities”, CRC Press, pp. 151-170, 2014.

[16] G. Giraldo, A. GabasIl, V. Nicoletti, J. Telis. "Propiedades termofísicas del jugo concentrado de lulo a temperaturas por encima del punto de congelación", Food Science and Technology, vol. 30, 2010.

[17] A. Albalasmeh, A. Asefaw, T, Ghezzehei. "A new method for rapid determination of carbohydrate and carbon concentrations using UV spectrophotometry", Carbohydrate Polymers, vol. 97, pp. 253-261, 2013. 